Revue d'histoire de l'Amérique française

7S REVUE D.HISTOIRE DE L'AMÉRIQUE FRANÇAISE

\title{
La population canadienne-française de l'Ouest des États-Unis. Un survol comparatif, 1850-1910
}

\section{Marie-Ève Harton et Danielle Gauvreau}

Volume 73, numéro 1-2, été-automne 2019

L’Ouest franco-américain

URI : https://id.erudit.org/iderudit/1068793ar

DOI : https://doi.org/10.7202/1068793ar

Aller au sommaire du numéro

\section{Éditeur(s)}

Institut d'histoire de l'Amérique française

\section{ISSN}

0035-2357 (imprimé)

1492-1383 (numérique)

Découvrir la revue

\section{Citer cet article}

Harton, M.-È. \& Gauvreau, D. (2019). La population canadienne-française de l'Ouest des États-Unis. Un survol comparatif, 1850-1910. Revue d'histoire de l'Amérique française, 73(1-2), 125-154. https://doi.org/10.7202/1068793ar
Résumé de l'article

On connaît peu de choses sur la population canadienne-française de l'Ouest américain, surtout avant que soit introduite une distinction entre les Canadiens français et les Canadiens anglais dans le recensement américain de 1890. Utilisant pour la première fois un dictionnaire des patronymes canadiens-français appliqué aux microdonnées des recensements américains, ce texte trace les grands traits de la population canadienne-française de l'Ouest américain entre 1850 et 1910 en la comparant à celle des autres régions des États-Unis. On y voit une population en croissance à la suite de l'arrivée de nouveaux immigrants, majoritairement des hommes, mais surtout en raison de l'augmentation du nombre de descendants d'immigrants. Au cours de cette période, des mouvements internes en provenance de l'Est sont aussi perceptibles, comme pour le reste de la population américaine. 


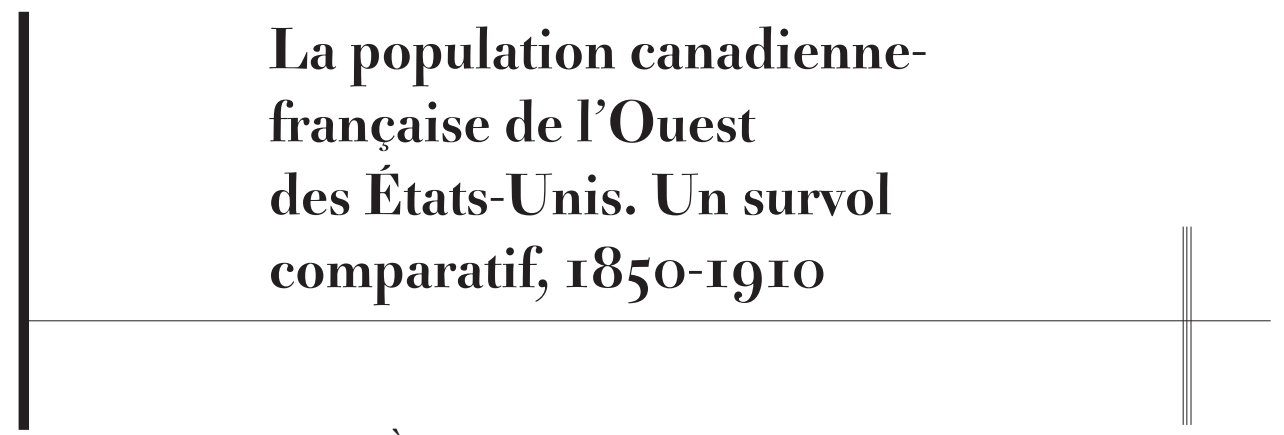

\author{
Marie-Ève Harton \\ Université du Québec à Trois-Rivières \\ Danielle GaUvreau \\ Université Concordial
}

\begin{abstract}
RÉsumé - On connaît peu de choses sur la population canadienne-française de l'Ouest américain, surtout avant que soit introduite une distinction entre les Canadiens français et les Canadiens anglais dans le recensement américain de 1890. Utilisant pour la première fois un dictionnaire des patronymes canadiens-français appliqué aux microdonnées des recensements américains, ce texte trace les grands traits de la population canadienne-française de l'Ouest américain entre 1850 et 1910 en la comparant à celle des autres régions des États-Unis. On y voit une population en croissance à la suite de l'arrivée de nouveaux immigrants, majoritairement des hommes, mais surtout en raison de l'augmentation du nombre de descendants d'immigrants. Au cours de cette période, des mouvements internes en provenance de l'Est sont aussi perceptibles, comme pour le reste de la population américaine.
\end{abstract}

1. Les auteures remercient Claude Leduc, assistant de recherche à l'Université Concordia, Laurent Richard, professionnel de recherche au Centre interuniversitaire d'études québécoises (CIEQ) à l'Université Laval, et Matt Nelson, analyste senior à l'Institute for Social Research and Data Innovation (ISRDI) à l'Université du Minnesota. Merci aussi à nos collègues Yves Frenette, Marc Saint-Hilaire et Hélène Vézina pour les échanges stimulants au fil de la création du Dictionnaire des patronymes ainsi qu'aux deux évaluateurs anonymes de la Revue pour leurs précieuses suggestions. L'appui financier du Conseil de recherches en sciences humaines du Canada (projet de développement de partenariat «Déploiements canadiens-français en Amérique du Nord (1760-1914)", dirigé par Y. Frenette et subvention Savoir pour un projet intitulé «Du Québec aux États-Unis. Nouvelle étude sociodémographique de l'émigration canadienne-française, 1850-1930", sous l'égide de D. Gauvreau) et celui du Fonds de recherche du Québec Société et Culture (Bourses de post-doctorat) ont rendu possible la réalisation de ce texte. 
ABstract - We know little about the French-Canadian population of the American West, especially before a distinction is added to the 1890 American census that allows us to distinguish French Canadians and English Canadians. Using for the first time a dictionary of French-Canadian surnames applied to American census microdata, this text draws the main features of the French-Canadian population of the American West from 1850 to 1910 by comparing it to other regions of the United States. It shows a growing population following the arrival of new immigrants, mostly men, but mainly due to the increase in the number of descendants of immigrants. Internal movements from the East also contribute to this growth during the period, as for the rest of the American population.

\section{INTRODUCTION}

De la Nouvelle-Angleterre au Midwest et à la Louisiane, en passant par la Californie, la présence canadienne-française peut être retracée sur l’ensemble du territoire américain depuis plusieurs siècles ${ }^{2}$. Les toponymes francophones, tout comme les nombreuses institutions sociales, religieuses et littéraires dans l'Ouest des États-Unis attestent la présence du fait français dans cette région depuis la fin du XVIII ${ }^{\mathrm{e}}$ siècle $^{3}$. Certains cas notoires, comme celui de Prudent Beaudry parti de Sainte-Anne-desPlaines (Québec) pour s'établir à Los Angeles, dont il devient maire en $1874^{4}$, témoignent de l'empreinte qu'a laissée l'importante mobilité des Canadiens français à l'échelle continentale. À la manière d'autres populations pionnières, les Canadiens français s'établissent de plus en plus à l'ouest sur cet immense territoire tout au long du XIX siècle.

Pourtant, l'immigration canadienne-française, ses mouvements et les dynamiques de cette population au sud de la frontière ont surtout été étudiés dans leur ensemble ou plus spécifiquement pour la NouvelleAngleterre et le Midwest ${ }^{5}$. Très peu d'attention a été accordée à l'Ouest,

2. Yves Frenette, Étienne Rivard et Marc St-Hilaire (dir.), La francophonie nord-américaine (Québec, Les Presses de l’Université Laval, 2012).

3. Pour une cartographie des toponymes francophones en Amérique du Nord entre 1790 et 1850, voir Y. Frenette, É. Rivard et M. St-Hilaire, La francophonie..., p. 66-67.

4. Yves Frenette, avec la collaboration de Martin Pâquet, Brève histoire des Canadiens français (Montréal, Boréal, 1998), p. 82.

5. Ralph D. Vicero, «Immigration of French Canadians to New England. A Geographic Analysis», thèse de doctorat (géographie), Université du Wisconsin, 1968; Yolande Lavoie, L'émigration des Canadiens aux États-Unis de 1840 à 1930 (Québec, Conseil de la langue française, 1981); Albert Faucher, Gilles Paquet et Wayne Smith, «L'émigration des Canadiens français vers les États-Unis, 1790-1940: problématique et coups de sonde", L'Actualité économique, 59, 3 (1983), p. 423-453; Bruno Ramirez, Par monts et par vaux. Migrants canadiens-français et italiens dans l'économie nord-atlantique 1860-1914 (Montréal, Boréal, 1991); Yves Frenette, "Macroscopie et microscopie d'un mouvement migratoire: les Canadiens français à Lewiston au XIX ${ }^{\mathrm{e}}$ siècle», dans Yves Landry et collab. (dir.), Les chemins de la migration en Belgique et au 
c'est-à-dire à la région comprise entre le fleuve Mississippi et l'océan Pacifique, ce dont témoigne un récent bilan historiographique de la francophonie nord-américaine publié dans le Bulletin d'histoire politique en $2016^{6}$. En fait, hormis quelques travaux qui ont touché à cette question sans en faire un objet précis d'analyse tels que l'ouvrage de Bruno Ramirez La ruée vers le sud: migrations du Canada vers les États-Unis, 1840-1930 ou des études portant sur un sous-groupe d'une région très ciblée comme celle d'Annick Foucrier sur les Français et les Canadiens français en Californie, l'historiographie ne comporte aucun portrait global traitant spécifiquement de la présence des Canadiens français dans l'Ouest ${ }^{8}$.

La seconde moitié du XIX siècle constitue une période particulièrement creuse pour les études sociodémographiques de la population canadiennefrançaise aux États-Unis. L'ouvrage de Marcus L. Hansen et de John B. Brebner intitulé The Mingling of the Canadian and American Peoples ${ }^{9}$, qui retrace sur presque trois siècles la mobilité des populations canadienne et étatsunienne à l'échelle du continent, illustre les difficultés qu'il y a à cerner cette population avant la fin du XIX ${ }^{\mathrm{e}}$ siècle. Cette étude contient des références au fait «français» au tout début de la période étudiée, soit dès le XVII ${ }^{e}$ siècle. Ce terme cède le pas à celui de "Canadien » à partir de la seconde moitié du XIX ${ }^{\mathrm{e}}$ siècle pour ensuite faire place à celui de "Canadien français» au tournant du $\mathrm{XX}^{\mathrm{e}}$ siècle. Le même constat vaut pour l'ouvrage de Leon E. Truesdell, The Canadian Born in the United States $^{10}$, qui couvre la période de 1850 à 1930 et qui fournit des statistiques séparées sur les Canadiens français et les Canadiens anglais à partir de 1890 seulement.

D’où provient cette difficulté à identifier spécifiquement les Canadiens français aux États-Unis avant 1890 ? Elle découle de l’absence de distinction

Québec, XVII ${ }^{e}$-XX $X^{e}$ siècles (Louvain-la-Neuve, Éditions Académia/Publications MNH, 1995), p. 221-232; Yves Roby, "Partir pour les "États” », dans Serge Courville, Atlas historique du Québec. Population et territoire (Sainte-Foy, Les Presses de l’Université Laval, 1996), p. 121-130; Yves Roby, Les Franco-Américains de la Nouvelle-Angleterre. Rêve et réalités (Sillery, Septentrion, 2000); Jean Lamarre, Les Canadiens français du Michigan. Leur contribution dans le développement de la vallée de la Saginaw et de la péninsule du Keweenaw 1840-1914 (Sillery, Septentrion, 2000).

6. Jean Lamarre (dir.), «La francophonie nord-américaine: bilan historiographique et bibliographique", Bulletin d'histoire politique, 24, 2 (hiver 2016).

7. Annick Foucrier, «Français et Canadiens français en Californie», dans Y. Frenette, É. Rivard et M. St-Hilaire, La francophonie..., p. 173-180.

8. C'est notamment un élément mentionné dans la conclusion d'Annick Foucrier, «Français et Canadiens français...».

9. Marcus Lee Hansen et John Bartlett Brebner, The Mingling of the Canadian and American Peoples (New Haven, Yale University Press, 1940).

10. Leon E. Truesdell, The Canadian Born in the United States. An Analysis of the Statistics of the Canadian Element in the Population of the United States 1850 to 1930 (New Haven, Yale University Press, 1943). 
faite entre les individus nés au Canada d'ascendance anglaise et ceux d'ascendance française dans les recensements américains de 1850 à $1880^{11}$. Cela ne permet conséquemment de circonscrire la population canadienne que dans son ensemble. C'est au recensement de 1890 que cette distinction apparait pour la première fois. Elle est ensuite reprise dans les recensements suivants. Toutefois, puisque les feuillets des manuscrits du recensement de 1890 ont été incendiés dans les années 1920, seuls les résultats déjà publiés sont désormais disponibles. L'exploitation des feuillets de recensement ou des nouvelles bases de données numérisées ne permet d'explorer le fait canadien-français qu'à partir de 1900, laissant dans l'ombre toute la seconde moitié du XIX siècle.

Plusieurs efforts de quantification par extrapolation, c'est-à-dire au moyen de mesures indirectes combinant l'information disponible dans plusieurs sources, ont néanmoins permis d'avoir une longue vue du phénomène d'émigration des Canadiens français vers les États-Unis au XIX ${ }^{\mathrm{e}}$ siècle. Les travaux de la démographe Yolande Lavoie ont permis d'estimer à 900000 le nombre total de personnes qui ont quitté le Québec pour aller aux États-Unis entre 1840 et $1930^{12}$. Un peu avant elle, l'économiste Gilles Paquet avait fait un tour d'horizon des défis méthodologiques posés par l'étude de l'émigration canadienne-française aux États-Unis et abordé la question des sources disponibles pour cerner le phénomène ${ }^{13}$. Il proposait alors une estimation globale du nombre de migrants canadiens-français dans chacune des régions américaines en 1870 et en 1880 . Sa méthode ne permet toutefois pas d'analyser les caractéristiques des individus qui composent cette population.

De manière générale, la plupart des études qui ont tracé des portraits sociodémographiques détaillés de la population canadienne-française au cours de la seconde moitié du XIX ${ }^{\mathrm{e}}$ siècle l'ont fait dans un cadre régional plus restreint, principalement en Nouvelle-Angleterre et dans le Midwest ${ }^{14}$.

11. Le premier recensement nominatif aux États-Unis a lieu en 1850. Les caractéristiques de toutes les personnes composant la population y sont colligées, pas seulement celles des ménages et de leurs chefs comme lors des dénombrements antérieurs (1790-1840). Pour une histoire de la pratique censitaire aux États-Unis, voir l'ouvrage de Paul Schor, Compter et classer. Histoire des recensements américains (Paris, Éditions de l'École des hautes études en sciences sociales, 2009).

12. L'ouvrage le plus cité à cet égard est celui de Y. Lavoie, L'émigration des Québécois... Le lecteur peut également consulter A. Faucher et al., «L'émigration des Canadiens français vers les États-Unis», p. 423-453 ainsi que Gilles Paquet «L'émigration des Canadiens français vers la Nouvelle-Angleterre, 1870-1910: prises de vue quantitatives ", Recherches sociographiques, 5, 3 (1964), p. 319-370.

13. G. Paquet «L'émigration des Canadiens français...».

14. R. D. Vicero, "Immigration of French Canadians...»; Frances Early, "The French-Canadian Beginnings in an American Community: Lowell Massachusetts, 1868-1886", thèse de doctorat (histoire), Université Concordia, 1979; Y. Frenette, «Macroscopie et microscopie... » ; Marie-Ève Harton, «Familles, 
Le défi était de taille, car la seule information contenue dans les recensements de 1850 à 1880 au sujet de l'origine des individus concerne le lieu de naissance et ces études reposent donc sur l'identification des Canadiens français sur la base de leurs patronymes. Qui plus est, jusqu'à la mise sur pied de l'importante infrastructure de recherche IPUMS ${ }^{15}$, l'identification des Canadiens français ne pouvait s'effectuer qu'au terme d'un long et fastidieux travail réalisé à partir des feuillets manuscrits disponibles sur microfilms. Le premier à appliquer cette méthode fut Ralph D. Vicero. Le géographe a ainsi dénombré manuellement toute la population canadienne-française en Nouvelle-Angleterre de 1840 à 1900. D’autres chercheurs ont appliqué une approche semblable à des corpus géographiques plus restreints et sur une période beaucoup plus courte ${ }^{16}$. L'utilisation des bases de microdonnées récemment mises à la disposition des chercheurs permet maintenant de préserver ces résultats et de reproduire l'opération d'identification des Canadiens français. Les récents travaux de Marie-Ève Harton sur la ville de Manchester ont mis en évidence l'intérêt de rendre pérenne cette longue et fastidieuse opération d'identification ${ }^{17}$.

Dans le cadre de ce numéro spécial portant sur l’Ouest franco-américain, cet article tente d'esquisser un premier portrait statistique détaillé de la population canadienne-française dans l'Ouest des États-Unis, du milieu du XIX ${ }^{\mathrm{e}}$ siècle (1850) jusqu'au début du XX $\mathrm{X}^{\mathrm{e}}$ siècle (1910). En plus de servir de toile de fond aux autres contributions de ce numéro, nous souhaitons que ce texte enrichisse l'historiographie du fait canadien-français au sud de la frontière canado-américaine en l'élargissant à un territoire peu étudié jusqu’à maintenant et à une période pour laquelle l’information sur la population canadienne-française fait cruellement défaut.

Le texte comporte deux parties. En raison de la difficulté posée par l'absence d'informations permettant d'identifier la population canadienne-

communautés et industrialisation en Amérique du Nord. Reproduction familiale canadienne-française à Québec et à Manchester (New Hampshire) au tournant du XX $\mathrm{XX}^{\mathrm{e}}$ siècle ", thèse de doctorat (sociologie), Université Laval, 2017 ; J. Lamarre, Les Canadiens français du Michigan...

15. Integrated Public Use of Microdata Series du Minnesota Population Centre de l'Université du Minnesota. Cette infrastructure de recherche rend accessibles depuis 2010 les microdonnées complètes de la population des États-Unis jusqu'en 1940. Minnesota Population Center. Integrated Public Use Microdata Series, International: Version 7.1 [dataset]. Minneapolis, MN: IPUMS, 2018. https://doi. org/10.18128/D020.V7.1 Pour plus d'informations, consulter la section «IPUMS USA» à: https://www. ipums.org/.

16. F. Early, "The French-Canadian Beginnings... »; Yves Frenette, «La genèse d'une communauté canadienne-française en Nouvelle-Angleterre: Lewiston, Maine, 1800-1880 ", thèse de doctorat (histoire), Université Laval, 1988; J. Lamarre, "Les Canadiens français du Michigan... ; M.-È. Harton, "Familles, communautés et industrialisation...».

17. M.-È. Harton, «Familles, communautés et industrialisation...». 
française dans les recensements américains avant 1890, nous consacrons une première section méthodologique à la présentation de notre approche - utilisée ici pour la première fois - et nous établissons certaines balises susceptibles de guider des travaux ultérieurs qui pourraient être menés avec le même outil. Dans un deuxième temps, nous présentons les premiers résultats d'analyses de la population canadienne-française dans l'Ouest américain à trois moments dans le temps, soit en 1850, en 1880 ainsi qu'en 1910. Sont alors examinés la composition de cette population en termes de générations (migrants ou descendants de migrants de $2^{\mathrm{e}}$ génération, $3^{\mathrm{e}}$ génération et suivantes) ainsi que les caractéristiques par âge et sexe, la répartition géographique et les lieux de naissance des individus qui la composent, particulièrement pour les Canadiens français résidant dans l'Ouest mais nés ailleurs aux États-Unis. Au fil du texte, des comparaisons sont effectuées avec les régions du Centre et de l'Est afin de mettre en exergue les particularités des populations canadiennes-françaises établies à l'ouest du Mississippi.

Cette approche diachronique pose le défi de la comparaison d'entités géographiques à travers le temps. Entre 1850 et 1910, la géographie politique des États-Unis change beaucoup, ce dont témoigne la variation du nombre d'États et de territoires au fil du temps. Dans cet article, nous avons opté pour une division de l'ensemble du territoire des États-Unis en fonction des barrières naturelles qu'ont dû surmonter les migrants qui se dirigeaient vers l'Ouest. La région de l'Ouest est donc définie comme recouvrant tout le territoire situé à l'ouest du Mississippi. Pour les fins de l'analyse, nous le comparons, d'une part, au territoire situé entre le Mississippi et les Appalaches que nous nommons le Centre, lequel englobe une bonne part du Midwest américain où d'importantes communautés canadiennes-françaises se sont implantées, et, d'autre part, à toute la Côte est (Est) qui comprend la Nouvelle-Angleterre, où se sont dirigés la majorité des Canadiens français durant le dernier tiers du XIX ${ }^{\mathrm{e}}$ siècle et le début du $\mathrm{XX}^{\mathrm{e}}$. Afin de faciliter la comparaison à travers le temps, ces distinctions ont été maintenues constantes pour l'analyse des trois recensements ${ }^{18}$.

18. Les fichiers de microdonnées obtenus dans IPUMS contiennent des informations géographiques standardisées. 


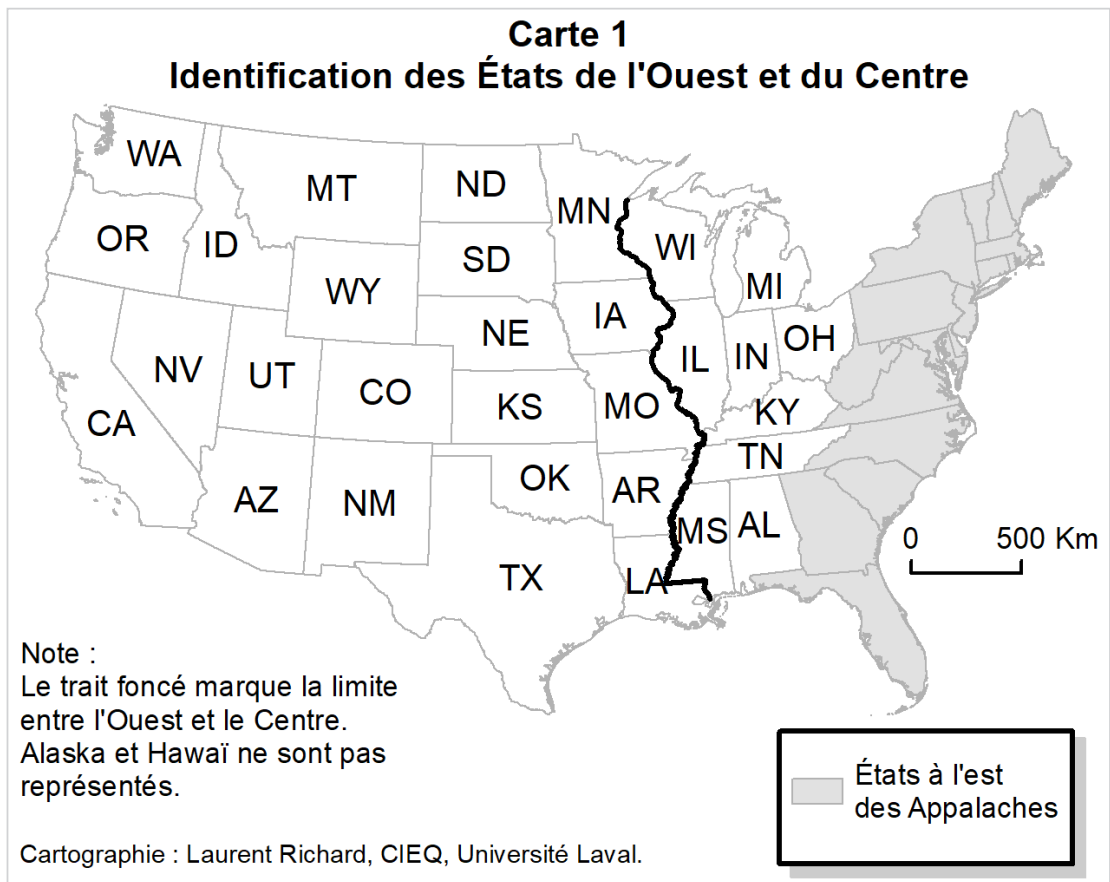

\section{NOUVEAUX CORPUS ET NOUVEL OUTIL POUR L'ÉTUDE DES CANADIENS FRANÇAIS DE 1850 À 1880}

\section{Les corpus de données}

Notre étude repose sur l'exploitation des microdonnées historiques des recensements américains disponibles à des fins de recherche par le biais de l'infrastructure de recherche IPUMS. Ces bases de microdonnées constituent de très vastes corpus numériques créés à partir de la saisie informatique des informations individuelles et nominatives tirées des fiches manuscrites des recensements. Celles-ci contiennent une manne d'informations pour l'étude détaillée des populations telles que l'âge, le sexe, le statut matrimonial, le lieu de naissance, la profession et la composition du ménage. Comparativement aux données agrégées publiées sous forme d'une série de tableaux croisés, l'exploitation des microdonnées permet la formulation de nouvelles questions de recherche pouvant être abordées sous divers angles, à l'aune de nouveaux paradigmes ainsi qu'au moyen d'un plus grand éventail de techniques statistiques.

Nous exploitons ici trois bases de microdonnées censitaires, soit celle de 1850 , créée à partir du tout premier recensement nominatif réalisé aux 
États-Unis, celle de 1880, puis celle de 1910. Le choix s'est fait de façon à permettre une observation de la population à des intervalles réguliers (30 ans) suffisamment espacés pour cerner l'évolution des dynamiques ainsi que pour des raisons pratiques visant à garder le nombre de bases de données à traiter dans des limites raisonnables.

Aux États-Unis, tout comme au Canada, les questions posées lors de l'énumération changent et leur nombre augmente considérablement d'un recensement à l'autre ${ }^{19}$. Les premières moutures du recensement américain, de 1850 à 1880, ne contiennent aucune information ayant trait à l'ethnicité ou à la langue, par exemple ${ }^{20}$. Le lieu de naissance des individus est toutefois noté, ce qui permet d'identifier les immigrants et leur pays d'origine. Plus spécifiquement, les individus nés à l'extérieur des États-Unis doivent indiquer leur pays de naissance, alors que les individus nés au pays doivent indiquer l'État dans lequel ils sont nés. À partir de 1880 s'ajoutent le lieu de naissance du père et celui de la mère, permettant ainsi d'identifier les enfants issus de l'immigration (génération 2 ) $^{21}$. L'autre changement important en ce qui concerne la population canadienne survient en 1890 , dans un contexte où les dirigeants politiques cherchent à mieux cerner les contours de l'immigration galopante. Comme le stipulent les instructions transmises aux énumérateurs, ceux-ci doivent désormais distinguer les individus nés au Canada selon qu'ils sont d'ascendance anglaise ou française au moyen des mentions «Canada English» ou «Canada French» pour chacun des individus nés au Canada ${ }^{22}$.

19. Pour les États-Unis, voir P. Schor, Compter et classer...; pour le Canada, Bruce Curtis, The Politics of Population. State Formation, Statistics, and the Census of Canada (Toronto, University of Toronto Press); Marie-Ève Harton, «Recensement et population canadienne-française. Catégories, références et identités", dans François Antaya, Marilyne Brisebois et Marie-Ève Harton, Regards interdisciplinaires sur le Québec. Actes des $17^{e}$ et $18^{e}$ colloques étudiants du CIEQ (Québec, Collection Cheminements, 2014), p. 33-42.

20. Des questions relatives à l'ethnicité et aux langues seront ajoutées par la suite dans le formulaire. Il est à noter qu'aucune question concernant la religion ne figure dans les recensements américains, historiques ou contemporains.

21. En 1870, les individus nés aux États-Unis devaient mentionner s'ils étaient nés de parents nés à l'étranger (oui ou non). En 1880, les autorités américaines posent deux questions «ouvertes» sur le lieu de naissance des parents de manière à élargir le spectre des connaissances en matière d'immigration.

22. Le Canada est le premier et le seul pays à faire l'objet d'une telle précision dans les instructions fournies aux agents énumérateurs en 1890. Les instructions se lisent comme suit : «If born in Canada or Newfoundland, write the word "English" or "French" after the particular place of birth, so as to distinguish between persons born in any part of British America of French and English extraction respectively. This is a most important requirement, and must be closely observed in each case and distinction carefully made» (Enumerator instructions, 1890 US Census). 


\section{Le Dictionnaire des patronymes canadiens-français et son application}

Le Dictionnaire des patronymes canadiens-français est constitué d'une liste de noms de famille à partir de laquelle il est possible d'identifier les Canadiens français, en l'absence d'autres informations pour le faire. Il consiste en une liste de patronymes recueillis sous leur forme standardisée, altérée ou anglicisée ${ }^{23}$. La création de cette liste a nécessité plusieurs étapes. Dans un premier temps, nous avons procédé à la collection du plus grand nombre possible d'altérations et d'anglicisations en utilisant comme point de départ une liste de patronymes standardisés provenant du fichier de population BALSAC de l'Université du Québec à Chicoutimi ${ }^{24}$. L'objectif était de pouvoir identifier les personnes dont le patronyme différait de sa forme standard. Par exemple, un agent énumérateur peut avoir inscrit «Jendreau» sur le feuillet d'énumération au lieu de "Gendreau» ou encore un dénommé «Boisvert» a pu traduire son nom en «Greenwood».

Dans un second temps, nous avons validé le Dictionnaire en l'appliquant au recensement américain de 1910 en sachant que l'information contenue dans ce recensement permet d'emblée d'identifier les Canadiens français. Cela fournit une base indépendante permettant d'évaluer la performance du Dictionnaire ${ }^{25}$. Deux groupes ont alors été comparés pour des fins de validation: 1) les Canadiens français identifiés par le Dictionnaire des patronymes $\left(\mathrm{CF}_{\mathrm{D}}\right)$ et 2$)$ les Canadiens français identifiés par les informations ayant trait aux lieux de naissance contenues dans le recensement $\left(\mathrm{CF}_{\mathrm{R}}\right)$. Les $\mathrm{CF}_{\mathrm{R}}$ sont des individus nés au Canada français ou dont au moins un parent est né au Canada français, selon les informations recueillies dans le recensement. Les $\mathrm{CF}_{\mathrm{R}}$ sont, par conséquent, tous de première ou de seconde génération. Afin de permettre la validation basée sur la comparaison des deux

23. Au total, cette liste comporte 11234 entrées. Pour toute information supplémentaire, communiquez par courriel avec les auteures (danielle.gauvreau@concordia.ca ou marie-eve.harton@uqtr.ca).

24. Amorcée dans le cadre du projet de développement de partenariat «Déploiements canadiensfrançais en Amérique du Nord (1760-1914)", la collection des altérations et des anglicisations s'est effectuée en collaboration avec les membres de la Société de généalogie de Québec. Ceux-ci furent invités à partager sur le site Web http://deploiements-francophones.ustboniface.ca/dictionnaire-des-patronyme/ toutes les mentions altérées et anglicisées rencontrées au fil de leurs recherches. L'outil a été finalisé dans le cadre du projet Savoir «Du Québec aux États-Unis. Nouvelle étude sociodémographique de l'émigration canadienne-française, 1850-1930». BALSAC est un fichier de population construit à partir des actes d'état civil du Québec pour l'ensemble des régions depuis les débuts du peuplement européen jusqu'à aujourd'hui. Pour plus d'information, consulter http:/ / balsac.uqac.ca/.

25. Le même travail aurait pu être réalisé avec le recensement de 1900, le premier pour lequel des microdonnées sont disponibles. Des raisons pratiques liées au fait que nous disposions déjà des données du recensement de 1910 dans le cadre d'un autre projet ont guidé ce choix. Nous poursuivons présentement des travaux méthodologiques complémentaires pour vérifier la stabilité des résultats obtenus avec le dictionnaire, mais nous voyons peu de raison que ce ne soit pas le cas. 
méthodes, les $\mathrm{CF}_{\mathrm{D}}$ sont donc ici des individus nés au Canada ou dont au moins un parent est né au Canada et qui portent un patronyme canadienfrançais. L'objectif des tests de validation consiste, d'une part, à mesurer la proportion de Canadiens français que le Dictionnaire permet d'identifier correctement par rapport à la définition du recensement (degré de couverture, que nous souhaitons le plus élevé possible) et, d'autre part, à mesurer la proportion d'individus identifiés par le Dictionnaire et qui ne sont pas d'origine canadienne-française au sens du recensement (degré d'imprécision, que nous souhaitons le plus faible possible).

Après plusieurs tests de validation portant sur différentes versions de la liste des patronymes, nous avons opté pour la version qui s’est révélée la plus performante, soit celle comprenant tous les patronymes portés par au moins $70 \%$ d'individus identifiés comme Canadien français dans le recensement de $1910\left(\mathrm{CF}_{\mathrm{R}}\right)$. Par exemple, si parmi tous les "Gagnon» recensés en 1910, plus de $70 \%$ sont nés au Canada français ou sont nés d'un père ou d'une mère nés au Canada français, le patronyme «Gagnon » a été retenu. Globalement, cette approche a permis de capturer un peu plus de $50 \%$ de tous les $\mathrm{CF}_{\mathrm{R}}$, un degré satisfaisant de couverture qui conduit en quelque sorte à disposer d'un échantillon d'un peu plus de la moitié de la population canadienne-française telle que définie par le recensement de 1910.

Qui sont ces Canadiens français d'après le recensement que le Dictionnaire ne parvient pas à cerner (des $\mathrm{CF}_{\mathrm{R}}$ qui ne sont pas $\left.\mathrm{CF}_{\mathrm{D}}\right)$ ? Il s'agit des porteurs de patronymes comptant moins de $70 \%$ de Canadiens français ${ }^{26}$ ainsi que des femmes canadiennes-françaises ayant épousé un non-Canadien français et leurs enfants, les premières parce qu'elles sont recensées sous le nom de leur époux et les enfants parce qu'ils portent le patronyme de leur père. Quant au degré d'imprécision, il se situe à $10 \%$ des individus identifiés par le Dictionnaire qui ne sont pas canadiens-français au sens du recensement (des $\mathrm{CF}_{\mathrm{D}}$ qui ne sont pas $\mathrm{CF}_{\mathrm{R}}$ ). En y regardant de plus près, cette imprécision trouve souvent sa source dans le fait que les mentions French ou English n'ont pas toujours été accolées au lieu de naissance «Canada» comme le requéraient les instructions aux énumérateurs ${ }^{27}$. L'imprécision provient donc alors du recensement et ces Canadiens français identifiés au moyen de la liste de patronymes seraient donc des Canadiens français que le recensement ne

26. C'est le cas notamment de patronymes comme "Martin», "Guy» ou encore «White» (anglicisation de "LeBlanc») qui peuvent tout autant correspondre à des individus anglophones que francophones.

27. Le nombre de ces cas n'est pas très élevé dans l'ensemble, mais il paraît plus important lorsqu'on le rapporte uniquement à la portion francophone du groupe. 
permet pas d'identifier. D’autres cas d'imprécision concernent des femmes non canadiennes-françaises portant le patronyme de leur mari canadien-français.

La couverture et la précision dans l'identification des Canadiens français au moyen de l'application du Dictionnaire varient d'une région à l'autre des États-Unis. C'est dans l'Ouest que le taux de couverture est le plus faible et le taux d'imprécision le plus élevé. Ce sont $39 \%$ des $\mathrm{CF}_{\mathrm{R}}$ qui sont saisis par le Dictionnaire et, parmi l'ensemble des Canadiens français identifiés par le Dictionnaire, environ le quart d'entre eux ne sont pas Canadien français au sens du recensement (imprécision). Les chiffres correspondants pour le Centre sont de $43 \%$ pour le degré de couverture et de $17 \%$ pour l'imprécision, tandis que dans l'Est le Dictionnaire est plus performant avec des taux de $56 \%$ et de $7 \%$ respectivement. Nous pensons que la performance moindre du Dictionnaire dans l'Ouest américain (et au Centre) pourrait découler de la plus faible proportion d'immigrants récents dans cette région comparativement à l'Est et d'une plus forte exogamie chez les Canadiens français en raison d'effectifs réduits, autant en nombres qu'en pourcentage de l'ensemble de la population de cette région ${ }^{28}$. L'imprécision des énumérateurs pourrait aussi y être plus répandue dans les endroits où la population canadienne est moins importante. Finalement, nous posons aussi l'hypothèse que les patronymes canadiens-français présents dans l'Ouest pourraient avoir subi davantage d'altérations qui ne sont pas prises en compte par le dictionnaire.

\section{Représentativité du corpus}

Même si le taux de couverture et le degré de précision varient d'une région à l'autre, le test le plus fiable pour valider l'application du dictionnaire des patronymes canadiens-français consiste à comparer les caractéristiques de la population canadienne-française définie à partir du dictionnaire $\left(\mathrm{CF}_{\mathrm{D}}\right)$ à celles de la population définie par le recensement $\left(\mathrm{CF}_{\mathrm{R}}\right)$. Chacune des régions est ici considérée séparément. Qui plus est, afin de tenir compte des pratiques d'énumération des femmes mariées, recensées sous le patronyme de leur mari, les résultats sont analysés séparément pour les hommes et pour les femmes.

Une plus grande proportion d'hommes que de femmes canadiens-français est saisie au moyen du Dictionnaire $\left(\mathrm{CF}_{\mathrm{D}}\right)$, comparativement à la définition

28. Ces facteurs structurels sont les premiers déterminants de la propension à contracter un mariage mixte. Voir par exemple, pour le Québec: Danielle Gauvreau et Patricia Thornton, «Marrying "the Other": Trends and Determinants of Culturally Mixed Marriages in Québec, 1880-1940", Canadian Ethnic Studies, 47, 23 (2015), p. 111-141. 
du recensement, et ce, surtout dans l'Ouest. Il en découle une certaine distorsion des rapports de masculinité lorsqu'on compare les deux méthodes d'identification des Canadiens français (Tableaux 1 et 2). La surestimation est de l'ordre de $21 \%$ dans l'Ouest, ce qui amplifie la sur-masculinité déjà présente dans cette région (119 hommes pour 100 femmes). La surestimation est moindre au Centre (12\%) et dans l'Est (3\%). À cet égard, le Dictionnaire est plus performant lorsqu'on en limite l'application aux seuls immigrants (génération 1 - résultats non montrés ici): le niveau de surestimation des rapports de masculinité diminue à $14 \%$ dans l'Ouest, à $6 \%$ au Centre et disparaît pratiquement dans l'Est (1\%). Un tel résultat paraît cohérent avec le fait que la propension des immigrants à l'exogamie est sans doute moindre que celle des Canadiens français de deuxième génération, d'une part parce que plusieurs immigrants arrivent déjà mariés et, d'autre part, parce qu'ils pourraient être plus nombreux à retourner épouser une femme dans le pays qu'ils viennent de quitter.

La comparaison des caractéristiques de la population canadienne-française définie par le recensement $\left(\mathrm{CF}_{\mathrm{R}}\right)$ et par le Dictionnaire $\left(\mathrm{CF}_{\mathrm{D}}\right)$ donne lieu à des résultats remarquablement similaires du côté des hommes (Tableau 1). Autant en ce qui a trait aux caractéristiques démographiques comme l'âge, l'état matrimonial ou même la génération (1 ou 2), qu’aux caractéristiques socioculturelles, comme le fait de savoir lire et écrire ou la capacité à parler anglais, les résultats sont quasi identiques. La même chose vaut pour la proportion vivant en milieu urbain ou rural. Bien que satisfaisants, les résultats de cette comparaison dans le cas des femmes présentent davantage d'écarts (Tableau 2). Comme on pouvait s'y attendre en raison du changement de patronyme des femmes mariées, le Dictionnaire identifie proportionnellement plus de jeunes femmes de moins de 15 ans qui sont célibataires; ces écarts sont généralement plus prononcés dans l'Ouest et au Centre, comparativement à l'Est où les mariages exogames seraient moins nombreux. Ces différences disparaissent presque complètement lorsqu'on restreint la comparaison aux femmes de première génération.

L'utilisation du Dictionnaire pour définir les contours de la population canadienne-française des États-Unis donne de très bons résultats, malgré quelques limitations dont il faudra tenir compte dans l'analyse. Ainsi, la population identifiée avec le Dictionnaire ne recouvre pas l'entièreté de la population canadienne-française, mais en constitue plutôt un échantillon de très bonne taille. Une certaine prudence s'impose donc dans l'interprétation des chiffres concernant la taille des populations, même si la cohérence interne de ces chiffres au fil du temps et au sein d'une même région paraît 
Tableau I

Distribution des Canadiens français au recensement de $1910\left(C_{R}\right)$ et selon le Dictionnaire des patronymes canadiens-français $\left(C_{D} F_{D}\right)$

\begin{tabular}{|c|c|c|c|c|c|c|}
\hline & \multicolumn{2}{|c|}{$\begin{array}{c}\text { Ouest } \\
\text { du Mississippi } \\
\text { (Ouest) }\end{array}$} & \multicolumn{2}{|c|}{$\begin{array}{c}\text { Du Mississipi } \\
\text { aux Appalaches } \\
\text { (Centre) }\end{array}$} & \multicolumn{2}{|c|}{$\begin{array}{c}\text { Est des } \\
\text { Appalaches (Est) }\end{array}$} \\
\hline & $\mathrm{CF}_{\mathrm{R}}$ & $\mathrm{CF}_{\mathrm{D}}$ & $\mathrm{CF}_{\mathrm{R}}$ & $\mathrm{CF}_{\mathrm{D}}$ & $\mathrm{CF}_{\mathrm{R}}$ & $\mathrm{CF}_{\mathrm{D}}$ \\
\hline \multicolumn{7}{|c|}{ Âge } \\
\hline $0-14$ & 22 & 23 & 25 & 27 & 32 & 34 \\
\hline $15-29$ & 27 & 28 & 28 & 28 & 29 & 29 \\
\hline $30-44$ & 24 & 24 & 21 & 21 & 21 & 20 \\
\hline $45-59$ & 18 & 17 & 16 & 15 & 12 & 12 \\
\hline $60-74$ & 7 & 7 & 7 & 7 & 5 & 4 \\
\hline $75+$ & 2 & 1 & 2 & 2 & 1 & 1 \\
\hline \multicolumn{7}{|c|}{ Statut matrimonial (15 ans + ) } \\
\hline Célibataire & 41 & 41 & 37 & 38 & 38 & 39 \\
\hline Marié & 53 & 53 & 57 & 57 & 57 & 57 \\
\hline Veuf & 5 & 5 & 5 & 4 & 5 & 4 \\
\hline Divorcé & 1 & 1 & 1 & 1 & 0 & 0 \\
\hline \multicolumn{7}{|c|}{ Génération } \\
\hline 1 & 35 & 35 & 33 & 34 & 44 & 45 \\
\hline 2 & 65 & 65 & 67 & 66 & 56 & 55 \\
\hline \multicolumn{7}{|c|}{$\%$ Sachant parler anglais (10 ans + ) } \\
\hline Oui & 99 & 98 & 98 & 98 & 89 & 88 \\
\hline \multicolumn{7}{|c|}{$\%$ Sachant lire et écrire $(10$ ans +$)$} \\
\hline Oui & 94 & 94 & 90 & 91 & 87 & 87 \\
\hline \multicolumn{7}{|c|}{$\%$ Vivant en milieu urbain } \\
\hline Urbain & 42 & 40 & 53 & 53 & 75 & 78 \\
\hline Nombre de cas & 58792 & 33582 & 81648 & 44056 & 351306 & 214143 \\
\hline $\begin{array}{l}\text { Rapport de } \\
\text { masculinité }\end{array}$ & 119 & 144 & 108 & 121 & 101 & 104 \\
\hline
\end{tabular}

Source: Recensement américain de 1910, Complete Count Data, IPUMS. 
Tableau 2

Distribution comparée des Canadiennes françaises au recensement de $1910\left(\mathrm{CF}_{\mathrm{R}}\right)$ et selon le Dictionnaire des patronymes $\left(\mathrm{CF}_{\mathrm{D}}\right)$

\begin{tabular}{|c|c|c|c|c|c|c|}
\hline & \multicolumn{2}{|c|}{$\begin{array}{l}\text { Ouest du } \\
\text { Mississippi } \\
\text { (Ouest) }\end{array}$} & \multicolumn{2}{|c|}{$\begin{array}{c}\text { Entre } \\
\text { Appalaches } \\
\text { et Mississippi } \\
\text { (Centre) }\end{array}$} & \multicolumn{2}{|c|}{$\begin{array}{c}\text { Est des } \\
\text { Appalaches } \\
\text { (Est) }\end{array}$} \\
\hline & $\mathrm{CF}_{\mathrm{R}}$ & $\mathrm{CF}_{\mathrm{D}}$ & $\mathrm{CF}_{\mathrm{R}}$ & $\mathrm{CF}_{\mathrm{D}}$ & $\mathrm{CF}_{\mathrm{R}}$ & $\mathrm{CF}_{\mathrm{D}}$ \\
\hline \multicolumn{7}{|c|}{ Âge } \\
\hline $0-14$ & 26 & 33 & 27 & 33 & 32 & 35 \\
\hline $15-29$ & 31 & 31 & 30 & 29 & 31 & 31 \\
\hline $30-44$ & 23 & 18 & 22 & 18 & 20 & 18 \\
\hline $45-59$ & 14 & 12 & 14 & 13 & 12 & 11 \\
\hline $60-74$ & 5 & 5 & 6 & 6 & 4 & 4 \\
\hline $75+$ & 1 & 1 & 1 & 1 & 1 & 1 \\
\hline \multicolumn{7}{|c|}{ Statut matrimonial $(15$ ans +$)$} \\
\hline Célibataire & 27 & 37 & 28 & 36 & 34 & 38 \\
\hline Mariée & 64 & 55 & 62 & 56 & 58 & 55 \\
\hline Veuve & 8 & 7 & 9 & 8 & 8 & 7 \\
\hline Divorcée & 1 & 1 & 1 & 0 & 0 & 0 \\
\hline \multicolumn{7}{|c|}{ Génération } \\
\hline 1 & 26 & 28 & 28 & 30 & 44 & 45 \\
\hline 2 & 74 & 72 & 72 & 70 & 56 & 55 \\
\hline \multicolumn{7}{|c|}{$\%$ Sachant parler anglais $(10$ ans + ) } \\
\hline Oui & 96 & 94 & 96 & 94 & 79 & 76 \\
\hline \multicolumn{7}{|c|}{$\%$ Sachant lire et écrire (10 ans + ) } \\
\hline Oui & 96 & 94 & 94 & 93 & 89 & 90 \\
\hline \multicolumn{7}{|c|}{$\%$ Vivant en milieu urbain } \\
\hline Urbain & 46 & 42 & 57 & 55 & 77 & 80 \\
\hline Nombre de cas & 49222 & 23390 & 75619 & 36382 & 349495 & 206631 \\
\hline
\end{tabular}

Source: Recensement américain de 1910, Complete Count Data, IPUMS. 
hautement probable. Puisque la proportion que représente cet échantillon varie d'une région à l'autre, il s'avère important d'utiliser le Dictionnaire à l'échelle régionale, plutôt que nationale, et il faut se garder d'amalgamer les résultats pour les États-Unis dans leur ensemble. Toutefois, nos tests de validation attestent de la robustesse de notre échantillon.

L'approche basée sur les patronymes que nous utilisons ici vise uniquement à délimiter les contours de la population canadienne-française au sein de la population américaine. Elle se distingue donc des études qui s'appuient sur la distribution géographique des patronymes comme marqueurs de la migration ${ }^{29}$. Nous avons plutôt recours dans le présent article aux variables du lieu de naissance et du lieu de naissance des parents disponibles dans les recensements pour analyser les parcours migratoires des Canadiens français installés dans l'Ouest, une fois ceux-ci identifiés par le Dictionnaire.

\section{LA POPULATION CANADIENNE-FRANÇAISE DANS LE FARWEST DES ÉTATS-UNIS}

Le tableau 3 présente les résultats de l'application du Dictionnaire de patronymes aux microdonnées des recensements américains de 1850, 1880 et 1910. À la différence des tests de validation qui ne portaient que sur les deux premières générations de Canadiens français, à des fins de comparaison avec l'information contenue dans le recensement, le Dictionnaire est maintenant appliqué à l'ensemble des porteurs de patronymes canadiens-français, qu'ils soient eux-mêmes nés au Canada ou issus de l'immigration canadienne-française plus ou moins récente. Les résultats sont présentés par génération et selon les informations disponibles à chaque recensement.

En 1850, l'absence d'informations concernant le lieu de naissance des parents fait en sorte que seuls les Canadiens français de la génération 1 peuvent être nettement distingués; tous les autres nés aux États-Unis et répondant aux critères du Dictionnaire sont regroupés dans une catégorie de deuxième génération et plus. Les recensements de 1880 et de 1910 permettent quant à eux de distinguer la seconde génération, c'est-à-dire les individus ayant au moins un parent né au Canada; c'est à partir de la troisième génération que l'ensemble des «autres» individus sont amalgamés.

29. Voir par exemple Michel Poulain, Michel Foulon, Anna Degioanni et Pierre Darlu, «Flemish Immigration in Wallonia and in France: Patronyms as Data", The History of the Family, 5, 2 (2000), p. 227241; Kevin Henry, «Exploring Population Structure and Migration with Surnames: Quebec, 1621-1900", thèse de doctorat (géographie), McGill University, 2004. 
Les chiffres concernant la première génération sont tout à fait comparables d'un recensement à l'autre. Il est important de préciser que le Dictionnaire permet, pour la première fois, de cerner un corpus de Canadiens français d'une grande profondeur généalogique. Bien sûr, nous savons que tous ces descendants de Gagnon, Beaudry ou Coutu s'identifient probablement à des degrés fort variés au groupe canadien-français, surtout à partir de la troisième génération. Le Dictionnaire offre néanmoins une base pour se pencher sur cette question dans des travaux ultérieurs.

En 1850, à l'ouest du Mississippi, les immigrants canadiens-français ne représentent que $1,8 \%$ de l'ensemble de la population canadienne-française de cette région ${ }^{30}$. Ce nombre pourrait être sous-estimé en raison de la grande mobilité liée aux activités économiques telles que le commerce des fourrures et la ruée vers l’or qui ont stimulé le déplacement plus à l'ouest. Au milieu du XIX ${ }^{\mathrm{e}}$ siècle, les Canadiens français présents dans l'Ouest sont donc presque tous nés aux États-Unis et ce constat vaut également pour le Centre et l'Est du pays où 2,9\% et 6,9\% de la population totale des Canadiens français sont des immigrants. Ces résultats étayent l'existence des liens très étroits entre les deux pays depuis plusieurs décennies et confortent l'idée que l'accroissement important de l'immigration canadienne-française au sud de la frontière se fait après la guerre de Sécession. C'est d'ailleurs ce dont témoigne la comparaison des effectifs en 1850 et 1880 . Le nombre et la proportion d'immigrants Canadiens français présents à l'ouest du Mississippi augmentent entre 1850 et 1880 , tant et si bien que la part des immigrants atteint $5,1 \%$. Ces proportions sont plus élevées dans les deux régions du Centre et de l'Est, où elles atteignent respectivement $8,6 \%$ et $19,8 \%$ en 1880 . Les centres industriels de la Nouvelle-Angleterre attirent une très grande partie de l'immigration canadienne-française à cette époque. Entre 1880 et 1910, l'Ouest continue d'attirer un bon nombre d'immigrants. Leur part relative décroît légèrement, en raison du poids de la seconde génération dans la population totale, un résultat tributaire du nombre d'arrivées au cours de la période précédente, de la croissance naturelle et d'un éventuel solde migratoire interne positif causé par l'afflux de population venant des autres régions des États-Unis, comme nous le verrons.

Une telle évolution paraît cohérente avec les données publiées à partir des recensements de 1890 et 1910 (Tableau 4). Ainsi, le ratio des effectifs des

30. En dépit d'un certain flou pouvant régner sur la définition de la frontière entre le Canada et les États-Unis dans cette région au milieu du XIX siècle et de son impact sur la déclaration du lieu de naissance des individus. 
Tableau 3

Population canadienne-française $\left(C_{D}\right)$ par génération et selon la région d'après l'application du Dictionnaire des patronymes canadiens-français, I850, 1880 et 1910.

\begin{tabular}{|c|c|c|c|c|c|c|}
\hline & \multicolumn{2}{|c|}{$\begin{array}{c}\text { Ouest du Mississippi } \\
\text { (Ouest) }\end{array}$} & \multicolumn{2}{|c|}{$\begin{array}{c}\text { Entre Appalaches } \\
\text { et Mississippi (Centre) }\end{array}$} & \multicolumn{2}{|c|}{$\begin{array}{l}\text { Est des Appalaches } \\
\text { (Est) }\end{array}$} \\
\hline \multicolumn{7}{|c|}{1850} \\
\hline Gen 1 & 690 & 1,8 & 2221 & 2,9 & 8260 & 6,1 \\
\hline Gen 2+ & 38195 & 98,2 & 75399 & 97,1 & 127692 & 93,9 \\
\hline Total & 38885 & 100,0 & 77620 & 100,0 & 135592 & 100,0 \\
\hline \multicolumn{7}{|c|}{1880} \\
\hline Gen 1 & 8788 & 5,1 & 17928 & 8,6 & 64530 & 19,8 \\
\hline Gen 2 & 10873 & 6,3 & 19609 & 9,5 & 43448 & 13,4 \\
\hline Gen 3+ & 151682 & 88,5 & 169738 & 81,9 & 217419 & 66,8 \\
\hline Total & 171343 & 100,0 & 207275 & 100,0 & 325397 & 100,0 \\
\hline \multicolumn{7}{|c|}{1910} \\
\hline Gen 1 & 18352 & 4,2 & 25601 & 6,8 & 189425 & 22,5 \\
\hline Gen 2 & 38620 & 8,8 & 54837 & 14,6 & 231349 & 27,5 \\
\hline Gen 3+ & 383080 & 87,0 & 294752 & 78,6 & 420750 & 50,0 \\
\hline Total & 440052 & 100,0 & 375190 & 100,0 & 841524 & 100,0 \\
\hline
\end{tabular}

Source: Recensements américains de I850, 1880 et 1910, Complete Count Data, IPUMS.

générations 1 et 2 est très similaire dans les deux tableaux, tout comme l'évolution régionale des effectifs entre 1890 (ou 1880) et 1910. Le Dictionnaire a l'avantage d'élargir la définition de Canadien français en englobant la troisième génération et les suivantes, un groupe qui forme déjà une très large sous-population à la fin du XIX ${ }^{e}$ siècle ${ }^{31}$. Les feuillets du recensement de 1890 étant désormais détruits, le recours au Dictionnaire paraît d'autant plus utile qu'il permet de combler l'absence d'informations sur l'ensemble des Canadiens français jusqu'au début du $\mathrm{XX}^{\mathrm{e}}$ siècle.

Les figures 1 et 2 rendent compte de la répartition géographique des effectifs de l'ensemble de la population canadienne-française ainsi que de celle des immigrants (première génération) vivant à l'ouest et au centre

31. Comme cette définition fondée sur le Dictionnaire est maintenant intégrée aux bases de données existantes, plusieurs nouvelles analyses sur des sous-groupes aux contours variables deviennent désormais possibles (jeunes, groupes socioprofessionnels, personnes âgées, etc.). 
Tableau 4

Population canadienne-française par génération et selon la région, d'après la définition du recensement, 1890 et 1910

\begin{tabular}{|l|c|c|c|c|c|c|}
\hline & $\begin{array}{c}\text { Ouest du Mississippi } \\
\text { (Ouest) }\end{array}$ & $\begin{array}{c}\text { Entre Appalaches } \\
\text { et Mississippi (Centre) }\end{array}$ & \multicolumn{2}{c|}{$\begin{array}{c}\text { Est des Appalaches } \\
\text { (Est) }\end{array}$} \\
\hline \multicolumn{7}{|c|}{1890} \\
\hline Gen 1 & 25945 & 49,2 & 46913 & 50,2 & 229638 & 59,0 \\
\hline Gen 2* & 26842 & 50,8 & 46544 & 49,8 & 159619 & 41,0 \\
\hline Total & $\mathbf{5 2 7 8 7}$ & $\mathbf{1 0 0}$ & $\mathbf{9 3 4 5 7}$ & $\mathbf{1 0 0}$ & $\mathbf{3 8 9 2 5 7}$ & $\mathbf{1 0 0}$ \\
\hline \multicolumn{7}{|c|}{} \\
\hline Gen 1 & 32207 & 31,4 & 46945 & 31,1 & 305931 & 44,1 \\
\hline Gen 2* & 70459 & 68,6 & 103909 & 68,9 & 388341 & 55,9 \\
\hline Total & $\mathbf{1 0 2} \mathbf{6 6 6}$ & $\mathbf{1 0 0}$ & $\mathbf{1 5 0 8 5 4}$ & $\mathbf{1 0 0}$ & $\mathbf{6 9 4 2 7 2}$ & $\mathbf{1 0 0}$ \\
\hline
\end{tabular}

Source: Calculs faits à partir de L. Truesdell, The Canadian Born..., p. 77-8I.

* Dans les données publiées par L.Truesdell, la génération 2 n'inclut pas les enfants dont seulement la mère est canadienne-française.

du pays ${ }^{32}$. Au début de la période étudiée, les Canadiens français de toutes les générations résident principalement dans les États de l’Est dont la frontière est délimitée à l'est par le Mississippi (Figure 1). Très peu sont recensés au centre de la région, mais ils sont tout de même présents dès 1850 à l'extrémité ouest des États-Unis (Californie et Oregon) ainsi qu'au sud (Nouveau Mexique et Texas). Au fil du temps, ils s'installent comme les autres populations dans l'ensemble du territoire à l'ouest du Mississippi et demeurent principalement présents aux abords du Mississippi et du Pacifique. Au cours de cet intervalle, la population totale recensée dans les États et territoires situés à l'ouest du Mississippi passe de 9,8 millions en 1850 à 27 millions en 1880, puis à 54,4 millions en 1910. Bien que la population canadienne-française représente moins de $1 \%$ de la population totale de l'Ouest, sa part relative augmente au fil du temps (de 0,4 à $0,8 \%$ ). Dit autrement, cela témoigne du fait que leur croissance relative dans l'Ouest du pays est plus importante que celle de la population totale. Par exemple, entre 1850 et 1880 , période pour laquelle la croissance relative de la population totale est plus importante que pour la période allant de 1880 à 1910 , la population totale de l'Ouest augmente de $173 \%$, alors que la population canadienne-française augmente de $340 \%$.

32. La région située à l'est des Appalaches n’est pas représentée sur les cartes. La croissance de ses effectifs y est telle que l'uniformisation de l'échelle cartographique devient quasi impossible. 
Les immigrants canadiens-français se distinguent par une présence plus massive dans le nord de la région, notamment dans les États limitrophes du Minnesota, du Dakota du Nord, du Montana et de l'Idaho ainsi que sur la Côte ouest. Ils semblent ainsi délaisser d'anciens itinéraires migratoires qui les conduisaient vers l'Iowa, le Missouri, l'Arkansas et la Louisiane. Les descendants des cohortes précédentes continuent pour leur part d'être très présents dans ces derniers États. Selon le tableau 5 , les immigrants sont surreprésentés en milieu urbain par rapport aux autres générations, particulièrement en 1910. Il s'agit là également d'un trait très fréquent des populations immigrantes canadiennes-françaises en Nouvelle-Angleterre ${ }^{33}$.

Les figures 3 à 5 représentent des pyramides des âges des immigrants canadiens-français (génération 1) pour l'Ouest et les deux régions de comparaison, aux trois moments des recensements. Celles-ci sont très différentes des pyramides des générations 2 et plus qui présentent une forme classique pour l'époque, soit une base élargie, une forme triangulaire rétrécissant avec l'âge et un équilibre général des sexes (non montrées ici). La figure 3 révèle la composition des populations qui ont forgé ces mouvements migratoires dans l'Ouest. Compte tenu des motivations des immigrants et des occasions d'emploi dans cette région, les pyramides traduisent un fort déséquilibre des sexes en faveur des hommes ${ }^{34}$. On dénote néanmoins un petit sursaut du côté féminin chez les jeunes adultes, peut-être le fait de couples d'immigrants, quoique la proportion non négligeable de célibataires parmi celles-ci laisse croire qu'elles s'y rendent, en partie du moins, pour occuper des emplois en support aux activités masculines (domestiques, cuisinières, etc.). La sur-masculinité des effectifs immigrants n'est pas spécifique à l'Ouest en 1850, mais elle y est beaucoup plus systématique à tous les âges et y perdure dans le temps, tandis qu'elle s'estompe progressivement au Centre (Figure 4) et s'inverse à l'Est à certains âges avec l'attrait qu'exerce l'industrie textile chez les femmes, jeunes et mariées (Figure 5). Dans l'Ouest, comme dans les autres régions, la migration familiale avec des enfants est perceptible à partir des pyramides des âges en 1850 et en 1880 . Elle est cependant beaucoup moins apparente en 1910, ce qui pourrait tenir au ralentissement de ce type de migration au profit de celle d'individus ou

33. François Weil, Les Franco-Américains, 1860-1980 (Paris, Belin, 1989).

34. On se rappellera que le déséquilibre des sexes en faveur des hommes est accentué par l'utilisation du Dictionnaire, mais pas au point de faire disparaître ce trait évident de l'immigration canadiennefrançaise vers l'Ouest. 


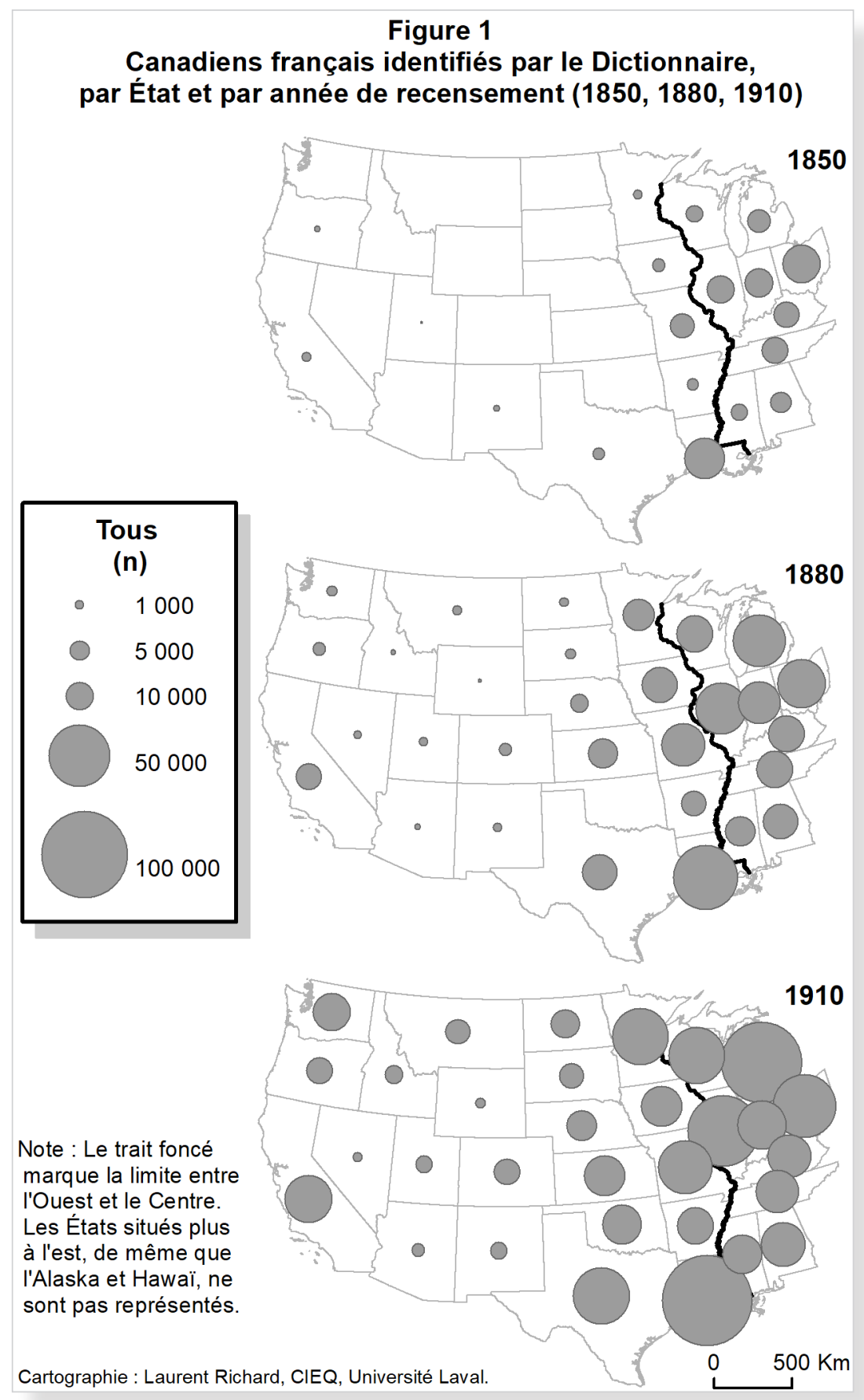

Source: Recensements américains de 1850, 1880 et 1910, Complete Count Data, IPUMS. 


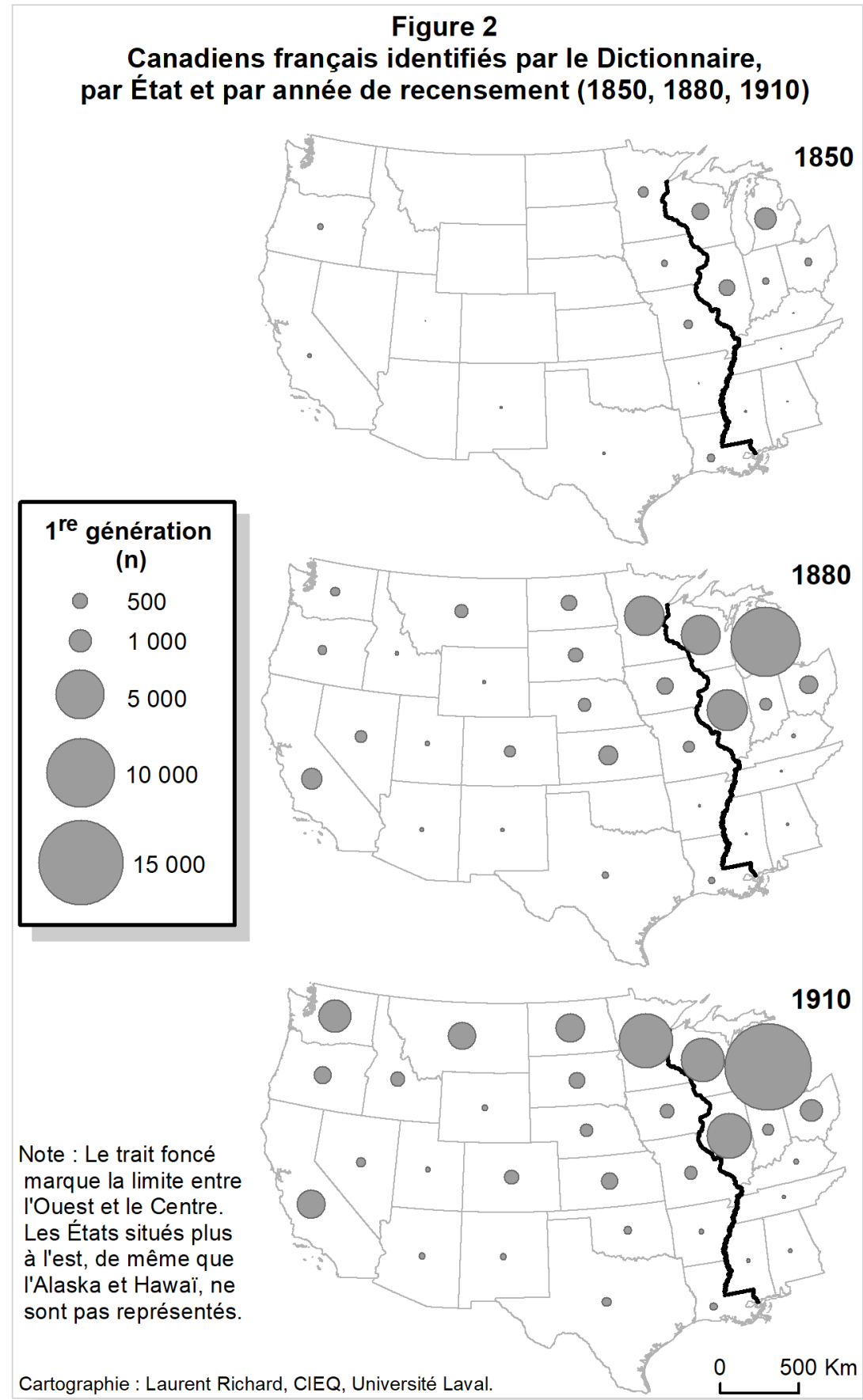

Source: Recensements américains de 1850, 1880 et 1910, Complete Count Data, IPUMS. 
Tableau 5

Milieu de résidence des Canadiens français dans l'Ouest des États-Unis selon la génération, I850, I880 et 1910

\begin{tabular}{|l|c|c|c|}
\hline & 1850 & 1880 & 1910 \\
\hline \multicolumn{4}{|c|}{ Immigrants (Gen1) } \\
\hline Rural & $84,5 \%$ & $82,1 \%$ & $54,2 \%$ \\
\hline Urbain & $15,5 \%$ & $17,9 \%$ & $45,8 \%$ \\
\hline \multicolumn{4}{|c|}{ Autres générations } \\
\hline Rural & $88,8 \%$ & $83,3 \%$ & $68,7 \%$ \\
\hline Urbain & $11,2 \%$ & $16,7 \%$ & $31,3 \%$ \\
\hline
\end{tabular}

Source: Recensements américains de 1850, 1880 et 1910, Complete Count Data, IPUMS.

de couples sans enfant. Les enfants arrivés plus tôt seraient déjà devenus de jeunes adultes dans l'intervalle.

L'information concernant le lieu de naissance est plus détaillée pour les individus nés aux États-Unis. Ceux-ci doivent déclarer dans quel État ils sont nés, ce qui permet d'examiner l'itinéraire des Canadiens français qui, à l'instar de leurs compatriotes américains et immigrants d'autres origines, ont tenté l'aventure et sont partis vers l'Ouest. Les Canadiens français de la deuxième génération recensés dans l'Ouest sont plus souvent nés dans une autre région que ne le sont les Canadiens français de troisième génération ou plus. Peut-être est-ce dû à une certaine forme de reproduction de la migration au sein des familles: les enfants de migrants pourraient avoir une plus grande propension à migrer eux-mêmes, autant parce que cette expérience migratoire fait partie de leur héritage familial que parce que leur réseau de connaissances s'étend plus souvent au-delà de leur lieu de résidence immédiat (Tableau 6). Parmi ces migrants «inter-régionaux », plus nombreux sont ceux qui arrivent du centre que de l'est du pays, ce qui suggère une migration secondaire de "proximité». Par exemple, les enfants nés de parents canadiens-français qui ont choisi de s'installer dans le Midwest, attirés par les terres, le travail en forêt et les mines à partir des années 1830, suivent le déplacement de ces activités à l'ouest du Mississippi. Les résultats montrent par ailleurs qu'entre 1880 et 1910, une part croissante d'individus de deuxième et de troisième générations sont natifs de l'Ouest, dans des proportions passant de $70 \%$ à un peu plus de $80 \%$. Bien que les tendances soient généralement les mêmes chez les femmes et chez les hommes, les premières sont un peu plus nombreuses 
Figure 3

Pyramides des âges de la population immigrante canadienne-française dans l'Ouest des États-Unis, I850, I880 et 1910

Immigrants canadiens-français, Région Ouest, 1850 ( $\mathbf{N}=690)$

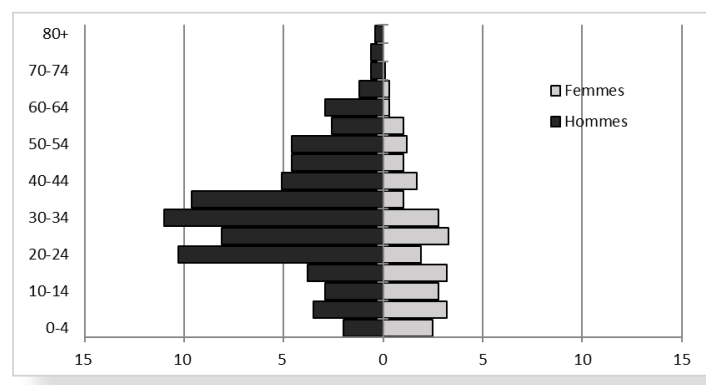

Immigrants canadiens-français, Région Ouest, I $880(\mathbf{N}=8788)$

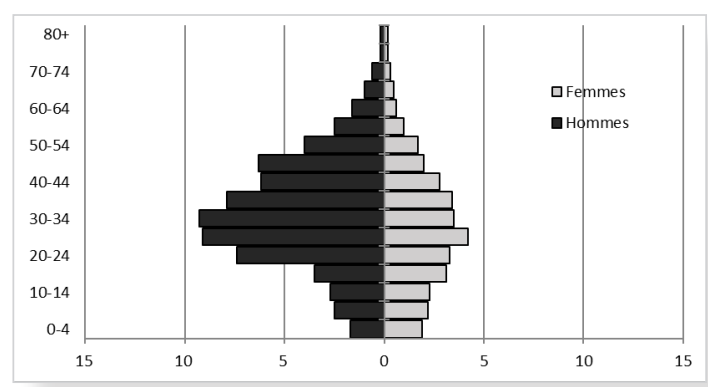

Immigrants canadiens-français, Région Ouest, I 910 ( $N=18$ 429)

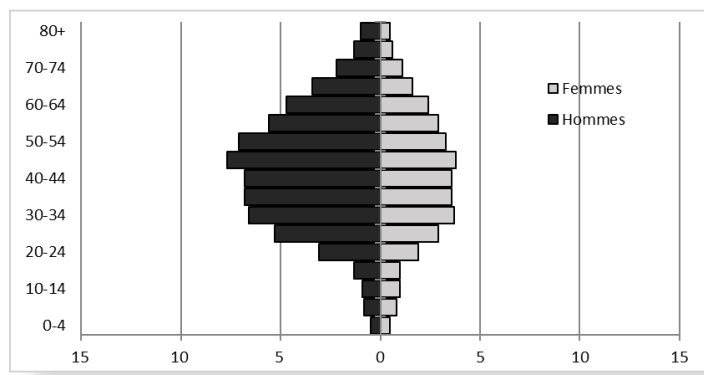


Figure 4

Pyramides des âges de la population immigrante canadienne-française dans le Centre des États-Unis, I850, 1880 et 1910

Immigrants canadiens-français, Région Centre, I 850 ( $\mathbf{N}=222$ I)

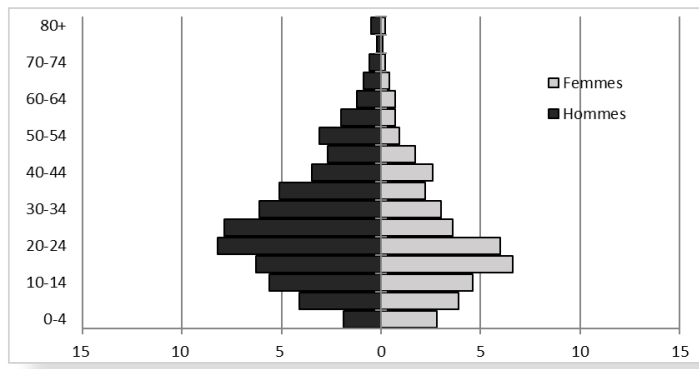

Immigrants canadiens-français, Région Centre, I 880 ( $N=17928)$

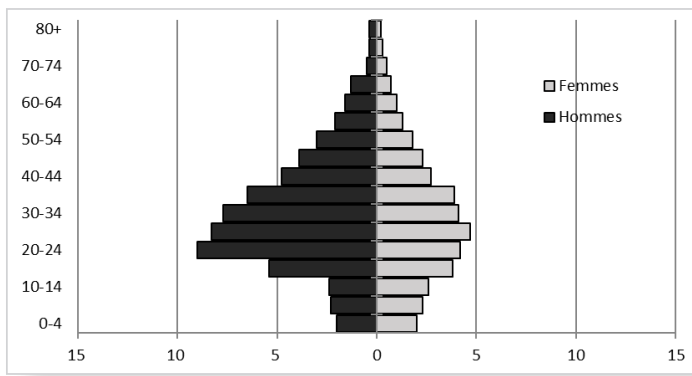

Immigrants canadiens-français, Région Centre, I 10 ( $\mathbf{N}=25$ 650)

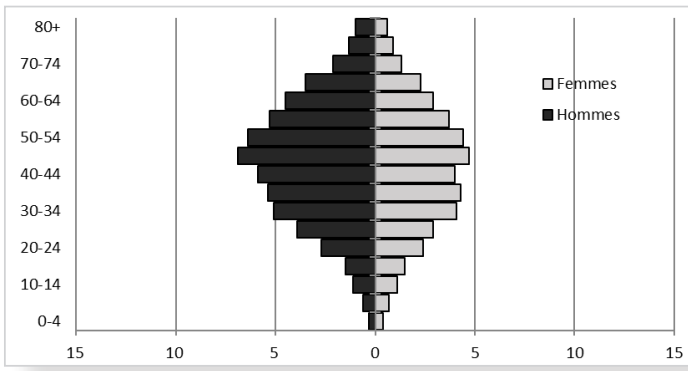




\section{Figure 5}

Pyramides des âges de la population immigrante canadienne-française dans l'Est des États-Unis, I850, 1880 et 1910

Immigrants canadiens-français, Région Est, 1850 ( $\mathbf{N}=8260)$

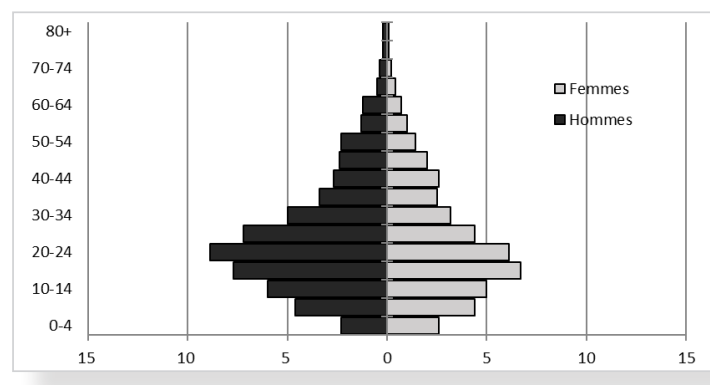

Immigrants canadiens-français, Région Est, I 880 ( $\mathbf{N}=64530)$

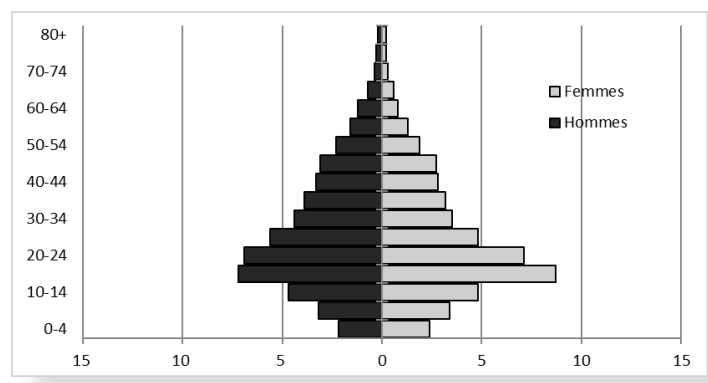

Immigrants canadiens-français, Région Est, I 910 ( $N=\mid 89$ 867)

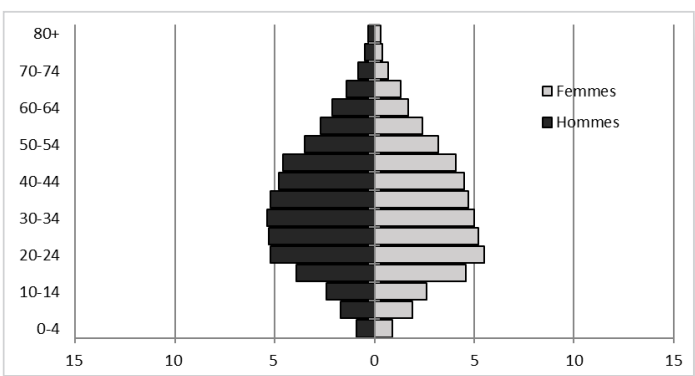


à être natives de l'Ouest que les hommes. L'attrait de l'Ouest en 1910 semble être un peu plus souvent l'affaire des hommes.

Le pourcentage de familles canadiennes-françaises résidant dans l'Ouest qui comptent des enfants nés dans l'une ou l'autre des deux autres régions du pays ou encore au Canada est loin d'être négligeable, et ce, autant chez les immigrants que parmi les Canadiens français de deuxième génération ${ }^{35}$ (Tableau 7). En 1850, 15\% des chefs de ménages canadiens-français de deuxième génération et plus ont au moins un enfant né dans le centre des États-Unis, comparativement à $5 \%$ seulement des immigrants (génération 1). Les chiffres suggèrent qu'une migration de type familial du centre vers l'ouest avait lieu au milieu du XIX ${ }^{\mathrm{e}}$ siècle et qu'elle est plus importante chez les descendants des Canadiens français (génération 2) que chez les immigrants, ce qui témoigne de cas de migrations secondaires entre les deux régions. En 1880 et en 1910, aucune différence n'est perceptible en ce qui a trait aux passages du centre vers l'ouest: la première et la deuxième génération le font dans d'égales proportions. C'est d'ailleurs en 1880 que la proportion d'individus qui sont recensés dans l'Ouest et dont un enfant est né dans le centre du pays est la plus élevée. Cela concorde avec le fort attrait exercé par le Midwest tout juste après la guerre de Sécession. La part de Canadiens français qui ont un enfant né dans l'Est est beaucoup moins importante et, autant en 1880 qu'en 1910, ce pourcentage est plus élevé chez les migrants canadiens-français que chez leurs compatriotes de seconde génération ( $10 \%$ et $6 \%$ en $1880,4 \%$ et $2 \%$ en 1910).

La période allant de 1880 à 1910 semble être une époque de grande transformation dans la mobilité des Canadiens français à l'échelle des États-Unis. La proportion de Canadiens français de l'Ouest qui ont des enfants nés soit dans le Centre ou dans l'Est diminue considérablement. Tant et si bien qu'en 1910, seulement $16 \%$ des migrants et $13 \%$ des enfants de migrants ont séjourné à l'est du Mississippi (Centre et Est ensemble). Le développement des transports, notamment les chemins de fer et les voies navigables, a-t-il facilité ou du moins raccourci le chemin

35. Cette approche se situe dans la foulée méthodologique d'études ayant analysé le phénomène de retours de Canadiens français en provenance des États-Unis, à partir des lieux de résidence des enfants indiqués dans les recensements canadiens: Martine Rodrigue, "Les Franco-Américains à Montréal en 1901: un regard sur le retour au pays», Francophonies d'Amérique, 9 (1999), p. 107-115 ; Paul-André Linteau, "Les migrants américains et franco-américains au Québec, 1792-1940. Un état de la question", Revue d'histoire de l'Amérique française, 53, 4 (2000) p. 561-602; Danielle Gauvreau, «Des États-Unis au Québec. Exploration du phénomène des migrations de retour des Canadiens français à partir des recensements canadiens ", communication présentée aux Journées d'études Déploiements canadiens-français en Amérique du Nord (1760-1914), Winnipeg, juin 2018. 
Tableau 6

\section{Région de naissance des Canadiens français de deuxième et de troisième générations et plus selon le sexe, individus recensés dans l'Ouest des États-Unis, I880, 1910}

\begin{tabular}{|c|c|c|}
\hline \multicolumn{3}{|c|}{1880} \\
\hline Homme né & $2 \mathrm{G}$ & $3 \mathrm{G}+$ \\
\hline Est & $12,1 \%$ & $11,0 \%$ \\
\hline Centre & $18,5 \%$ & $14,5 \%$ \\
\hline Ouest & $69,4 \%$ & $74,5 \%$ \\
\hline \multicolumn{3}{|c|}{1910} \\
\hline Homme né & $2 \mathrm{G}$ & $3 \mathrm{G}+$ \\
\hline Est & $8,7 \%$ & $5,6 \%$ \\
\hline Centre & $17,5 \%$ & $11,4 \%$ \\
\hline Ouest & $72,4 \%$ & $82,2 \%$ \\
\hline \multicolumn{3}{|c|}{1880} \\
\hline Femme née & $2 \mathrm{G}$ & $3 \mathrm{G}+$ \\
\hline Est & $9,1 \%$ & $8,9 \%$ \\
\hline Centre & $17,5 \%$ & $13,5 \%$ \\
\hline Ouest & $73,3 \%$ & $77,6 \%$ \\
\hline \multicolumn{3}{|c|}{1910} \\
\hline Femme née & $2 \mathrm{G}$ & $3 \mathrm{G}+$ \\
\hline Est & $5,2 \%$ & $4,3 \%$ \\
\hline Centre & $14,4 \%$ & $10,3 \%$ \\
\hline Ouest & $79,2 \%$ & $84,8 \%$ \\
\hline
\end{tabular}

Source: Recensements américains de 1850, 1880 et 1910, Complete Count Data, IPUMS.

** Pourcentages manquants $=$ modalités de réponses qui n'ont pas pu être classées, ex. USA, unknown, etc.

vers l'Ouest? Ces passages se faisaient-ils davantage, au début du $\mathrm{XX}^{\mathrm{e}}$ siècle, à un âge où les individus n'ont pas encore fondé de famille? Ces deux hypothèses apparaissent très plausibles.

Par ailleurs, un peu plus d'une famille d'immigrants canadiens-français sur cinq en 1850 migre dans l'Ouest avec au moins un enfant né au Canada. Ce chiffre se maintient en 1880, mais décline à $13 \%$ seulement en 1910. Ce constat est conséquent avec la diminution de la part que représentent les jeunes dans les pyramides des âges à cette dernière date. Cela reflète sans doute les variations du calendrier d'arrivée et les carac- 
Tableau 7

Mobilité des familles avec enfants attestée par la présence d'au moins un enfant né ailleurs que dans l'Ouest du pays selon la génération du chef de ménage, I850, I880, 1910

\begin{tabular}{|c|c|c|}
\hline \multicolumn{3}{|c|}{1850} \\
\hline Au moins un enfant né... & Immigrants & Génération 2+ \\
\hline Est & $5 \%$ & $5 \%$ \\
\hline Centre & $5 \%$ & $15 \%$ \\
\hline Canada & $22 \%$ & $0 \%$ \\
\hline \multicolumn{3}{|c|}{1880} \\
\hline Au moins un enfant né... & Immigrants & Génération 2 \\
\hline Est & $10 \%$ & $6 \%$ \\
\hline Centre & $19 \%$ & $19 \%$ \\
\hline Canada & $24 \%$ & $2 \%$ \\
\hline \multicolumn{3}{|c|}{1910} \\
\hline Au moins un enfant né... & Immigrants & Génération 2 \\
\hline Est & $4 \%$ & $2 \%$ \\
\hline Centre & $12 \%$ & $11 \%$ \\
\hline Canada & $13 \%$ & $1 \%$ \\
\hline
\end{tabular}

Source: Recensements américains de 1850, 1880 et 1910, Complete Count Data, IPUMS.

téristiques des migrants au début du $\mathrm{XX}^{\mathrm{e}}$ siècle ${ }^{36}$. Même si le pourcentage de Canadiens français de deuxième génération ayant au moins un enfant né au Canada en 1880 et en 1910 est faible, il témoigne de l'existence d'allers-retours entre le Canada et les États-Unis, malgré la distance. C’est là une des formes de maintien des liens entre les communautés de part et d'autre de la frontière, comme l'ont notamment attesté les correspondances étudiées par plusieurs chercheurs ${ }^{37}$.

\section{CONCLUSION}

La création du Dictionnaire des patronymes canadiens-français permet enfin de tracer les contours de la population canadienne-française aux

36. Bruno Ramirez (avec la collaboration d’Yves Otis), La Ruée vers le Sud. Migrations du Canada vers les États-Unis, 1840-1930 (Boréal, Montréal, 2003).

37. Yves Frenette, Marcel Martel et John Willis, Envoyer et recevoir. Lettres et correspondances dans les diasporas canadiennes (Québec, Les Presses de l’Université Laval, 2006); Mario Mimeault, L'exode québécois, 1852-1925: correspondances d'une famille dispersée d'Amérique (Québec, Septentrion, 2013). 
États-Unis durant la seconde moitié du XIX ${ }^{\mathrm{e}}$ siècle. Pendant longtemps, l'absence de question directe sur les caractéristiques ethniques ou linguistiques des individus dans les recensements américains a limité l'analyse de ce groupe. Exploitant les patronymes des individus auxquels les chercheurs ont désormais accès dans les récentes bases de microdonnées des recensements historiques (IPUMS, Université du Minnesota), le Dictionnaire des patronymes canadiens-français permet maintenant d'identifier, de manière automatisée, un important échantillon de Canadiens français. Nos tests montrent que cet échantillon se révèle représentatif de la population totale canadienne-française. Moyennant quelques réserves concernant la taille exacte des groupes, et certaines précautions concernant les caractéristiques de la population féminine au-delà de la première génération, le portrait qui en découle peut néanmoins être qualifié de fort riche. Dans l'Ouest américain, l'application du Dictionnaire a permis de tracer ici les grandes lignes du fait canadienfrançais dans cette région, phénomène qui, à grande échelle, est encore mal connu. Il s'agit là d'une percée importante dans l'historiographie.

La population canadienne-française de l'Ouest augmente considérablement entre 1850 et 1910 et l'information relative aux immigrants (génération 1) montre une accélération de ce mouvement entre 1850 et 1880 , suivie d'un certain ralentissement entre 1880 et 1910. L’Ouest bénéficie cependant de mouvements en provenance de l'est des États-Unis et sa population canadienne-française surpasse celle de la région située entre les Appalaches et le Mississippi en 1910. L'immigration canadienne-française vers l'Ouest présente un fort déséquilibre en faveur des hommes, un trait qui ne se dément pas au fil du temps, contrairement à ce qui se passe dans les régions du Centre et, surtout, de l'Est. Les différentes régions des ÉtatsUnis attirent donc des populations aux traits contrastés, et cela vaut aussi pour la population canadienne-française.

Ce premier survol descriptif de la population canadienne-française dans l'Ouest américain entre 1850 et 1910 ne constitue qu'un premier pas. À la différence des méthodes employées dans les études antérieures pour identifier les Canadiens français à partir des images des manuscrits, le Dictionnaire des patronymes canadiens-français est un outil pérenne, accessible électroniquement et applicable à des ensembles de données de tailles variables et même de sources diverses. Nous souhaitons qu'il donne un souffle nouveau aux travaux sur la population canadienne-française aux États-Unis et qu'il inspire peut-être des travaux sur d'autres communautés issues de l'immigration aux États-Unis au cours de la même 
période. Même si le lieu de naissance suffit généralement pour identifier une population immigrante, par exemple en provenance d'Italie ou des Pays-Bas, l'approche préconisée ici pourrait être utilisée dans d'autres cas où une population nationale présente des divisions identifiables par les patronymes (la Flandre et la Wallonie en Belgique, par exemple) ou encore afin d'identifier les descendants de migrants aux États-Unis avant qu'une variable concernant le lieu de naissance des parents ne devienne disponible en 1880. À cet égard, il importe de souligner que la notion de génération au sein de la population canadienne-française aux États-Unis s'est avérée cruciale pour comprendre la composition et la dynamique de cette population dont la vaste majorité est composée, déjà en 1850, de descendants de deuxième génération et plus. Cette étude trace ainsi de manière originale un pont entre les migrations du XVIII ${ }^{e}$ siècle et l'intensification des migrations au cours de la seconde moitié du XIX ${ }^{\mathrm{e}}$ siècle.

Le présent article s'est penché sur un nombre limité de caractéristiques des individus et des ménages puisque nous avons privilégié une longue vue fondée sur l'exploitation de trois recensements pour lesquels la quantité et le type d'information disponibles varient beaucoup. Plusieurs pistes s'offrent maintenant aux chercheurs qui s'intéressent à la population canadienne-française: des travaux plus poussés sur les immigrants canadiens-français et leurs descendants dans certaines régions encore plus circonscrites des États-Unis et à des moments spécifiques; des études plus approfondies sur les porteurs de patronymes canadiens-français de troisième génération et plus afin d'étudier leur degré d'appartenance à la culture canadienne-française ; des études ciblées visant à jumeler les immigrants en provenance du Québec au fichier de population BALSAC afin de lier les informations dans la société de départ et au lieu de destination; une analyse plus fine d'ensembles plus restreints couplée à d'autres sources de nature qualitative. À l'instar d'autres travaux de nature historique sur les migrations, nous sommes d'avis que l'étude de la mobilité des Canadiens français contribue à alimenter notre compréhension plus large des mouvements migratoires ainsi qu'à parfaire notre connaissance de l'histoire de la population canadienne-française en général. 